\title{
CONSTRUÇÃO DE MODELO MATEMÁTICO REGRESSIVO PARA RACIONALIZAÇÃO DOS TEMPOS DE PROCESSAMENTO DAS ATIVIDADES DE PRÉ-IMPRESSÃO DE UMA GRÁFICA
}

\section{CONSTRUCTION OF MATHEMATICAL MODELS RETURN FOR RATIONALIZATION OF THE PROCESSING TIME OF ACTIVITIES FOR PRE-PRINTING OF GRAPHIC}

\author{
Marcio Carvalho da Silva ${ }^{1}$; Maria de Lourdes Gomes Barreto ${ }^{2}$; Luiz Bueno da Silva ${ }^{3}$ \\ ${ }^{1}$ Universidade Federal da Paraíba - UFPB - João Pessoa - Brasil \\ mkarvalho2005@yahoo.com.br \\ ${ }^{2}$ Universidade Federal da Paraíba - UFPB - João Pessoa - Brasil \\ marilu@ct.ufpb.br \\ ${ }^{3}$ Universidade Federal da Paraíba - UFPB - João Pessoa - Brasil \\ bueno@ct.ufpb.br
}

\begin{abstract}
Resumo
O presente artigo aborda um estudo realizado no departamento de Pré-impressão (PI) de uma gráfica, com o objetivo de verificar a influência da variável tempo de processamento das atividades desenvolvidas no departamento para a preparação das chapas de impressão. Os resultados poderão fornecer subsídios para tomada de decisão. Para tanto, utilizou-se gráfico de pareto $e$ modelagem multivariada a partir do tratamento de 30 ordens de produção. Concluiu-se que cerca de $49 \%$ do tempo total de processamento está vinculado a gargalos existentes no sistema; e 96,4\% da variação do tempo improdutivo foram devidas às ociosidades existentes durante a preparação das chapas de impressão.
\end{abstract}

Palavras-chave: análise de regressão, tempo de processamento, pré-impressão.

\section{Introdução}

Com o advento dos princípios da qualidade Total em atendimento às exigências do mercado, tornou-se mais acirrada a busca por melhoria contínua e, consequentemente, a perseguição por sistemas mais eficazes em termos de confiabilidade, rapidez, flexibilidade, custos, entre outros fatores que contribuem para a redução dos índices de falhas tendendo a níveis próximos de zero.

Embora o interesse maior seja de manter os padrões nos sistemas produtivos visando a redução dos fatores que geram improdutividade nos sistemas, é razoável admitir que eles ocorram independente de qual seja a sua procedência. Isso porque não existe um sistema infalível capaz de 
assegurar um processo que ocorra 100\% livre de erros, caso contrário seria dispensável os esforços dos departamentos de qualidade dentro das organizações.

Os fatores que geram improdutividade nos processos produtivos devem ser controlados sob pena de que se tenham elevados índices nos custos da produção. De acordo com Almeida Filho et al (2008), o conhecimento desta improdutividade e seu impacto no tempo total de fabricação são fundamentais, pois abre caminho para um planejamento buscando racionalizar a produção.

O presente artigo tem por objetivo analisar a relação dos tempos improdutivos das atividades do departamento de pré-impressão de uma gráfica na preparação de chapas de impressão.

\section{Tempos Improdutivos em processos customizados}

De acordo com Filho (2008), customização é a estratégia de oferecer aos clientes o fornecimento de produtos diferenciados ou individualizados, com o maior número de opções possíveis. Essa estratégia, segundo Machado (2008), pode ser compreendida por um conjunto de planos que irão guiar os serviços para alcançar os objetivos de agilidade, eficiência e preços similares aos dos produtos em massa.

No entanto, verifica-se que os produtos com características customizadas, como é o caso dos produtos do setor gráfico, apresentam uma variedade de procedimentos inerentes a cada um dos serviços solicitados pelos clientes e, com isso, favorece a geração ou aumento dos tempos improdutivos por serem atividades que exigem constante aprovação dos clientes e inúmeros ajustes nos arquivos antes de serem definitivamente produzidos em grande escala.

Slack et al (2002) classifica esses tipos de processos como processos de jobbing, isto é, são aqueles que em geral apresentam alta variedade e baixo volume de produção, onde os produtos compartilham os mesmos recursos de operação (pessoas e máquinas) com diversos outros produtos, exigindo o mesmo tipo de atenção, mas diferindo entre si pelas necessidades exatas que cada um apresenta.

É importante frisar que, embora se faça uso de máquinas e equipamentos comuns nos seus processos de fabricação, a programação dessas máquinas exige do operador uma atenção redobrada para evitar a ocorrência de falhas pelo ajuste inadequado nos arquivos dos clientes, e consequentemente elevação nos tempos de processamento das atividades, visto que cada produto imputa procedimentos diversos e, por conseguinte, uma forte tendência à ocorrência de erros.

Por esta razão, observa-se que as organizações desenvolvem técnicas de gestão que objetivam reduzir essas disfunções e, para tanto, faz uso das mais diversas formas de abordagem dos problemas, como é o caso da utilização das ferramentas da qualidade na detecção de disfunções nos processos produtivos, princípios da engenharia de métodos no que tange ao estudo das 
atividades laborais em que pese a maneira como são executadas as atividades visando atingir maiores níveis de produtividade pelo uso racional dos fatores de produção, e métodos estatísticos no que consiste em confrontar variáveis de decisão visando identificar aquelas com maior grau de significância, isto é, identificar as variáveis que mais influenciam no aumento dos tempos improdutivos. Tais ferramenta e métodos servem de instrumentos promotores de decisões mais assertivas que respaldem os gestores na implementação de melhorias justificando, assim, os investimentos despendidos nesse processo.

\section{Gerenciamento de tempos improdutivos}

Os ganhos de produtividade são necessariamente atingidos quando são eliminados ou reduzidos os desperdícios dentro da organização. Partindo desse princípio que sustenta a filosofia JIT (Just in Time), muitas organizações têm analisado seus sistemas produtivos a fim de identificar pontos críticos passíveis de melhorias para garantirem aumento da eficiência produtiva através da racionalização de seus recursos e, assim poderem se manter competitivas no mercado. O controle dos tempos de produção tem sido um desses pontos principais, em particular, o tempo considerado improdutivo provocado, na maioria das vezes, por falta de adequado acompanhamento e planejamento da rotina de trabalho.

Para Gaither e Frazier (2008), o modo como os processos são gerenciados desempenha um papel fundamental na melhoria da produtividade. Isso significa que o gerenciamento eficaz se caracteriza pelo controle de medidas múltiplas de desempenho, estabelecendo metas para o futuro e buscando melhores maneiras de criar e operar processos.

De acordo com Ohno (1997), a verdadeira melhoria na eficiência surge quando produzimos zero desperdício e levamos a porcentagem de trabalho para $100 \%$. Isto significa dizer que ao manter o controle dos processos de fabricação de forma que se utilize de meios adequados para garantir um fluxo de informações e materiais sem a presença de fatores externos os quais figuram como empecilho ao processo, bem como ao reduzir o tempo ocioso do pessoal envolvido nas atividades de fabricação e dispor de um ambiente devidamente adequado às atividades de trabalho, torna-se possível atingir níveis de eficiência satisfatórios e, consequentemente, aumento na produtividade.

A Organização Internacional do Trabalho (OIT) aborda as questões voltadas ao tempo total de produção como sendo um composto entre o tempo efetivo de trabalho e o tempo desperdiçado com irregularidades do sistema, conforme apresentado no esquema da figura 1 . Verifica-se que o tempo total da operação nas condições atuais é dividido em regiões (A, B e C) onde para cada uma dessas regiões representa uma parcela do tempo improdutivo total que pode ser classificado como se segue. 
REGIÃO A $\rightarrow$ tempo destinado a realização de trabalho suplementar devido a métodos ineficazes;

REGIÃO B $\rightarrow$ tempo improdutivo devido a ações gerenciais mal desempenhadas;

REGIÃO C $\rightarrow$ tempo improdutivo provocado pelo mal desempenho dos funcionários.

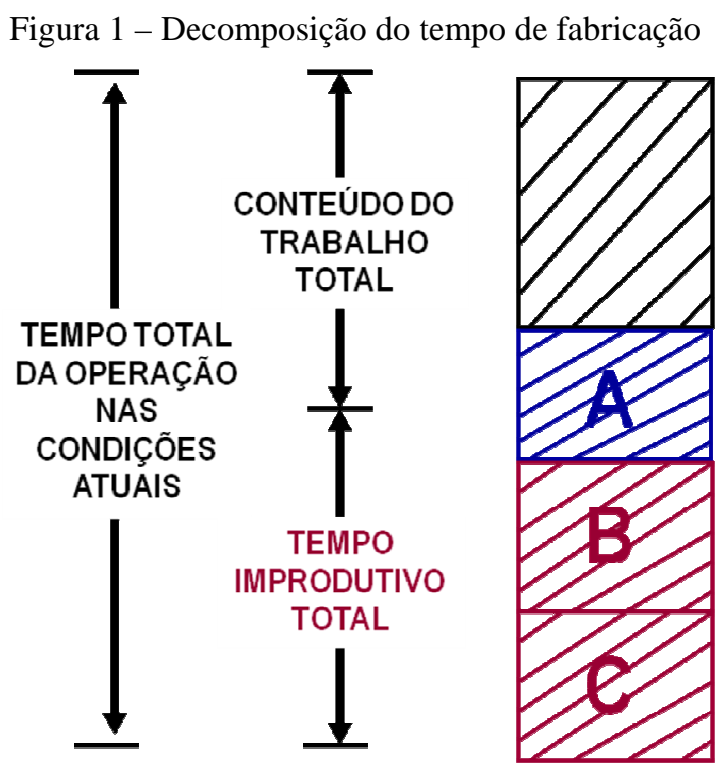

Fonte: Adaptado OIT (1994)

No sistema de pré-impressão gráfica, os problemas com tempos improdutivos são freqüentes, às vezes oriundos de atrasos na transmissão dos artigos do cliente para a empresa no momento em que lhe foi encaminhado para aprovação; outras vezes devido a irregularidades nas atividades internas do setor que promove a formação de filas de Ordens de Produção; ou ainda, por ineficiência no repasse de informações do departamento comercial da empresa no que tange ao detalhamento dos serviços solicitados pelo cliente.

Visando analisar a variabilidade do sistema da Pré-impressão, desenvolveu-se um método de controle dos tempos improdutivos permitindo identificar quais dos problemas ocorrem em maior frequiência e com isso, auxiliar os gerentes de produção em tomar medidas de resolução dos problemas de maneira mais segura, atuando nos pontos de efetiva relevância. O método consiste em coletar informações dos tempos de processamento das atividades de pré-impressão e analisar, à luz dos conceitos de regressão multivariada, quais das variáveis que mais contribuem para elevar o tempo improdutivo do processo. O método será melhor apresentado nas seções subseqüentes.

\section{Análise de regressão e o processo de tomada de decisão}

Segundo Aragão (2007) a verdadeira função da gestão de qualquer negócio está baseada na geração de resultados empresariais sustentáveis - o que possibilitará a sua sobrevivência, o seu 
crescimento e a sua perpetuidade. Cabe então às empresas modernas, definir e implantar uma sistemática associada a sua estratégia de negócio que possa identificar os principais pontos de perda e, contínua e consistentemente, buscar alternativas para eliminá-las ou reduzi-las, agregando valor a todas as partes interessadas, os stakeholders.

Nesse contexto, admite-se a criação de diversas metodologias com o objetivo de auxiliar os gestores no processo de tomada de decisão, tais como: controle estatístico de processos; utilização das ferramentas da qualidade; experimentos estatísticos (árvores de perdas, modelagens matemáticas), ciclo PDCA, entre outros.

O processo de tomada de decisão exige dos gerentes e responsáveis pela produção um conhecimento apurado do sistema produtivo como um todo, de tal forma que identifiquem os pontos mais vulneráveis à incidência de erros que provoquem alterações significativas no planejamento e programação da produção. Dessa forma, é muito comum recorrer aos métodos estatísticos, pois fornecem subsídios que respaldam as decisões mais coerentes.

Dentre os métodos estatísticos mais comumente utilizados na gestão do negócio, encontra-se a regressão linear (RL), pois traduz de maneira mais consistente os pontos de maior vulnerabilidade do sistema produtivo. A RL é a técnica utilizada para se estabelecer um modelo matemático que interprete a relação entre variáveis que estejam correlacionadas (PINCOLINI, 2004).

Através da RL é possível analisar os gargalos do processo produtivo, podendo ser implementado novas metodologias e tecnologias que venham a melhorar a qualidade do produto e garantir uma maior, ou melhor, produtividade dos profissionais envolvidos (SANTOS e JÚNIOR, 2003).

No presente artigo, utilizou-se da regressão múltipla para identificar as variáveis que contribuem para o aumento do tempo improdutivo nos processos de pré-impressão através do modelamento matemático que serviu de auxílio ao processo de tomada de decisão.

\section{Procedimentos Metodológicos}

O método científico para o desenvolvimento do presente artigo, no que tange a natureza da pesquisa, consiste em um estudo de caso realizado no departamento de Pré-impressão (PI) de uma gráfica, onde se buscou analisar os tempos de produção de cada atividade desenvolvida no departamento, no intuito de identificar os fatores que mais contribuem para o aumento do tempo improdutivo no setor.

Trata-se ainda de uma pesquisa de caráter quali-quantitativo uma vez que, além de pesquisa documental (abordagem qualitativa), utilizou-se de instrumentos de coleta de dados utilizados para a inferência estatística através da análise de regressão linear, que possibilitou verificar o 
comportamento existente entre as variáveis abordadas.

\subsection{Variáveis do estudo}

As variáveis analisadas para a construção do modelo matemático correspondem aos tempos improdutivos pela interrupção das atividades no setor de Pré-impressão durante a execução das ordens de produção (OP). As principais causas da interrupção foram codificadas afim de facilitar no preenchimento da ficha de coleta de dados. Cada código representa, portanto uma variável preditora que contribui no aumento do Tempo Improdutivo (variável resposta).

As atividades da PI são distribuídas em 4 funções, a saber: Montagem Eletrônica, Imposição Eletrônica, Gravação de chapa e Revelação da matriz de verniz. As três primeiras foram consideradas no estudo. A atividade de revelação da Matriz de verniz não foi considerada, haja vista ser uma atividade que não ocorre periodicamente, isto é, só é feita à medida que o cliente solicita a laminação no seu arquivo impresso.

Os tempos dessas atividades são classificados em três, que consistem na subdivisão do tempo total do processo:

- Tempo Total: é a diferença entre o tempo de início e de término de cada atividade;

- Tempo de Interrupção (Improdutivo): é a diferença entre o tempo de início e término de uma interrupção, caracterizado pelo registro de uma irregularidade no processo, do momento de sua ocorrência até o final da mesma, quando a atividade volta a seu fluxo normal de execução;

- Tempo Real: é o tempo destinado a execução da atividade, isto é, a diferença entre o tempo total e o tempo de interrupção.

O presente artigo, no entanto, concentrou-se em avaliar os tempos improdutivos ocasionados por interrupções que ocorrem no sistema.

\subsection{Instrumentos para coleta de dados}

Para a realização da coleta desses tempos, desenvolveu-se um formulário de controle de produção constando das informações inerentes ao serviço a ser executado. Os procedimentos para aplicação do formulário seguem descritos a seguir, conforme os critérios de: alimentação, preenchimento, recolhimento e tabulação de dados. 
- Quanto à alimentação do formulário: Esse procedimento é feito de duas formas dependendo do tipo de serviço estabelecido na ordem de produção (OP). No caso de serviços cujos arquivos são do tipo abertos, isto é, que permitem alterações pela Préimpressão, os formulários seguem o fluxo iniciando pela atividade de Montagem. No caso de arquivo do tipo fechado, isto é, arquivos que não podem ser alterados, o fluxo dos formulários inicia na atividade de imposição;

- Quanto à sequência de preenchimento do formulário: o formulário segue o fluxo normal de uma Ordem de Produção, sendo preenchido em cada etapa das atividades da PI pelos próprios funcionários do departamento, os quais o destinam às atividades subseqüentes até que percorra todo o processo e retorne completamente preenchido;

- Quanto ao recolhimento do formulário: essa etapa fica sob responsabilidade do gravador de chapa que, no início do primeiro turno, recolhe os formulários e confere as OPs liberadas para a Impressão através do relatório "Posição das OP abertas no PCP" contido no Sistema de Informação interno da empresa. Em seguida, entrega os formulários ao assistente de coordenação;

- Quanto à tabulação dos dados: os dados serão tabulados em planilhas eletrônicas que servirão posteriormente para alimentar o software de tratamento estatístico.

\section{Descrição do processo analisado}

O departamento de pré-impressão é responsável pelo fornecimento de chapas gravadas, ao Setor de Impressão. Essas chapas irão alimentar as impressoras planas funcionando como se fossem carimbos dos conteúdos do produto final, podendo ser imagens e/ou textos contidos em revistas, tablóides, livros, folders, entre outros produtos do portfólio da empresa. As impressoras planas reproduzem os arquivos no papel e encaminha ao Setor de Acabamento para finalização do produto.

O quadro a seguir apresenta as atividades desenvolvidas na Pré-impressão com suas respectivas descrições e características. Em seguida o processo é representado no macro-fluxograma (figura 2). 
Quadro 1 - Descrição das atividades da Pré-impressão

\begin{tabular}{|c|c|c|}
\hline $\begin{array}{l}\text { ATIVIDADES DA } \\
\text { PRÉ-IMPRESSÃO }\end{array}$ & DESCRIÇÃO & CARACTERÍSTICAS \\
\hline Montagem Eletrônica & $\begin{array}{l}\text { Gerar o plotter de baixa qualidade, montar o } \\
\text { layout, revisar, aplicar todos os carimbos e } \\
\text { informações necessárias; adequação de cores. }\end{array}$ & $\begin{array}{c}\text { - Aplicável para } \\
\text { arquivos do tipo } \\
\text { aberto (quando é } \\
\text { permitido edição); } \\
\text { - Se a aprovação pelo } \\
\text { cliente for por via } \\
\text { digital, criar arquivos } \\
\text { em formato JPEG }\end{array}$ \\
\hline Imposição Eletrônica & $\begin{array}{l}\text { Criação do template, importação da imposição, } \\
\text { importação dos arquivos dentro da imposição, } \\
\text { revisão digital da imposição, confecção e revisão } \\
\text { de layout e confecção de layout de verniz } \\
\text { reduzido } \rightarrow \text { Atividades de finalização }\end{array}$ & $\begin{array}{l}\text { Aplicável para arquivos } \\
\text { do tipo fechado (quando } \\
\text { não é permitido edição) }\end{array}$ \\
\hline Gravação de Chapas & $\begin{array}{l}\text { Gravação, controle de qualidade de gravação e } \\
\text { revelação, furação e dobra, envio de carga de tinta } \\
\text { aos equipamentos de impressão }\end{array}$ & $\begin{array}{l}\text { Aplicável a todos os } \\
\text { arquivos independente } \\
\text { de ser aberto ou } \\
\text { fechado }\end{array}$ \\
\hline $\begin{array}{c}\text { Montagem da matriz de } \\
\text { verniz }\end{array}$ & $\begin{array}{l}\text { Descarregar os laserfilmes e iniciar a preparação } \\
\text { das telas de verniz conforme o procedimento, } \\
\text { verificando o número de telas que serão } \\
\text { necessários para o serviço em questão }\end{array}$ & $\begin{array}{l}\text { Aplicável de acordo } \\
\text { com a solicitação do } \\
\text { cliente. Caso não seja } \\
\text { necessário, essa } \\
\text { atividade é suprimida. }\end{array}$ \\
\hline
\end{tabular}

Fonte: Autoria própria (2009)

Figura 2 - Macrofluxo dos processos da Pré-impressão

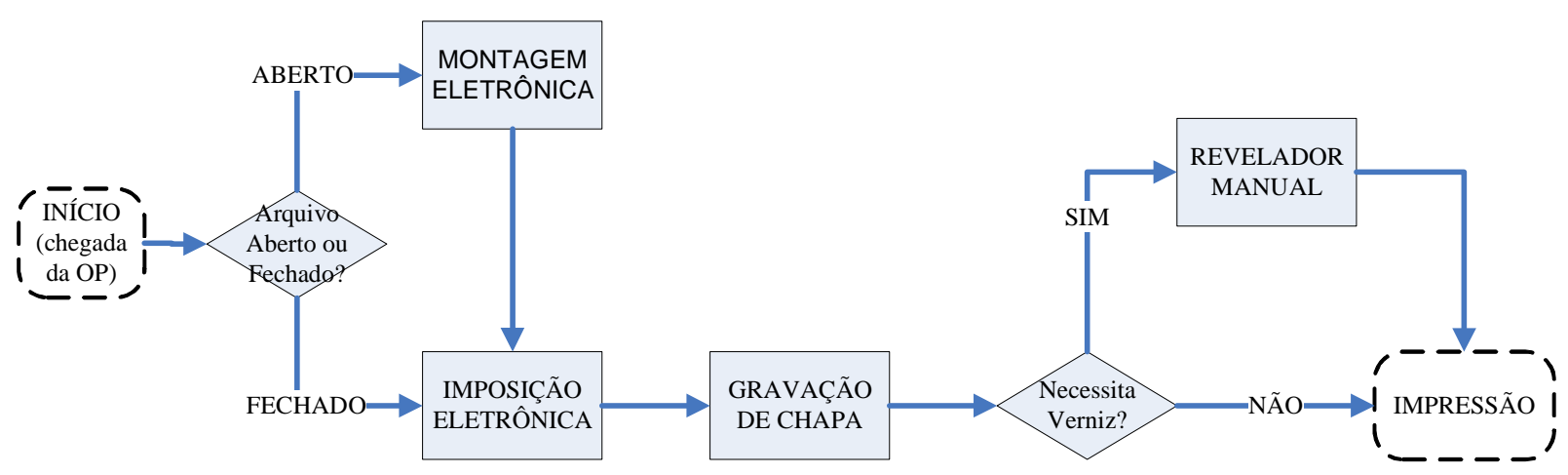

Fonte: Autoria própria (2009)

\section{Coleta de Dados - processo de medição}

Para realização do estudo de caso, foram analisadas 30 ordens de produção emitidas pelo PCP ao departamento de Pré-impressão totalizando 599,78 horas de produção.

O processo de medição foi realizado através de apontamento de horas indicados pelos próprios funcionários da PI, através de formulário de controle de tempos de processamento. Foram desenvolvidos códigos de improdutividade disponibilizados em folha de controle de tempos de processamento (figura 3). Tais códigos referem-se aos tempos improdutivos identificados durante as atividades do departamento (montagem, imposição e gravação). 
Figura 3 - Folha de controle de tempos de processamento

\begin{tabular}{|c|c|c|c|c|c|c|c|c|}
\hline \multicolumn{9}{|c|}{ CONTROLE DOS TEMPOS DE PROCESSAMENTO } \\
\hline \multirow[b]{2}{*}{ Data } & \multirow[b]{2}{*}{$\mathbf{N}^{\circ} \mathbf{O P}$} & \multicolumn{3}{|c|}{ Atividade } & \multicolumn{3}{|c|}{ Interrupções } & \multirow[b]{2}{*}{ OBSERVAÇÕES } \\
\hline & & $\begin{array}{c}\text { Tipo de } \\
\text { atividade }\end{array}$ & $\begin{array}{c}\text { Hora } \\
\text { Inicial } \\
\end{array}$ & Hora Final & $\begin{array}{c}\text { Hora } \\
\text { inicial }\end{array}$ & $\begin{array}{l}\text { Hora } \\
\text { Final } \\
\end{array}$ & Motivo & \\
\hline & & & $\ldots$ & - & -__- & $\ldots$ & & \\
\hline & & & $\ldots$ & $\ldots$ & $\ldots$ & $\ldots$ & & \\
\hline & & & $\ldots$ & _- & $\ldots$ & $\ldots$ & & \\
\hline & & & $\ldots$ & $\ldots$ & _-_- & $\ldots$ & & \\
\hline & & & $\ldots$ & $\ldots$ & $\ldots$ & - & & \\
\hline \multicolumn{9}{|c|}{ PRINCIPAIS MOTIVOS DE INTERRUPÇÕES } \\
\hline \multirow{2}{*}{\multicolumn{7}{|c|}{$\begin{array}{l}001 \text { - AGUARDANDO PROJETO DO DESENVOLVIMENTO } \\
002 \text { - PROBLEMAS NO FORMATO DO AROUIVO }\end{array}$}} & \multicolumn{2}{|c|}{009 - MÁQUINA EM MANUTENÇÃO } \\
\hline \multicolumn{6}{|c|}{002 - PROBLEMAS NO FORMATO DO ARQUIVO } & & \multicolumn{2}{|c|}{010 - AGUARD. LIBERAÇÃO DO ARQUIVO } \\
\hline \multicolumn{7}{|c|}{003 - AUSÊNCIA DO ARQUIVO DE VERNIZ } & \multicolumn{2}{|c|}{011 - ARQUIVOS PRIORITÁRIOS } \\
\hline \multicolumn{7}{|c|}{004 - PROBLEMAS DE PAGINAÇÃO } & \multicolumn{2}{|c|}{012 - REUNIÕES, TREINAMENTOS } \\
\hline \multicolumn{7}{|c|}{005 - PROBLEMAS COM A LOMBADA } & \multicolumn{2}{|c|}{013 - PROBLEMAS DE PROGRAMAÇÃO } \\
\hline \multicolumn{7}{|c|}{006 - AUSÊNCIA DE PROJETO } & \multicolumn{2}{|c|}{014 - SISTEMA DE INFORM. TRAVANDO } \\
\hline \multicolumn{7}{|c|}{007 - AGUARDANDO MODIFICAÇÕES NO ARQUIVO } & \multicolumn{2}{|c|}{015 - AGUARDANDO PLOTTER } \\
\hline \multicolumn{7}{|c|}{008 - DIVERGÊNCIA DE COR E FONTE } & \multicolumn{2}{|c|}{016 - OUTROS PROBLEMAS } \\
\hline
\end{tabular}

Fonte: Autoria própria (2009)

A partir dos dados registrados na folha de controle, obteve-se os valores dos tempos totais de cada uma das atividades do setor da PI (tabela 1). Cada número de OP representa um tipo de serviço realizado pela PI, por exemplo, OP1 refere-se a um serviço, no caso Folder, medido nas atividades de montagem (5,96 horas), Imposição (8,95 horas) e gravação de chapas (3,22 horas) totalizando 18,12 horas que este serviço levou até ser totalmente produzido para ser liberado ao processo de impressão. Vale ressaltar que o tempo foi totalizado desde o momento que a OP deu entrada no setor de PI até o momento de sua saída para o setor de impressão. Mais adiante será mostrado que destas 18,12 h uma parte foi destinada ao tempo produtivo (TP) inerente às atividades do departamento e a outra parte foi consumida por atividades improdutivas (TI), ou seja, manobras, recuperações, esperas entre outras atividades que caracterizaram interrupções no processo. 
Tabela 1 - Tempos totais por OP

\begin{tabular}{|c|c|c|c|c|c|}
\hline \multicolumn{2}{|c|}{$\underset{\rightarrow}{\text { PROCESSOS }}$} & Montagem & Imposição & Gravação & \multirow{2}{*}{ TOTAL } \\
\hline OP & Serviço & Tempo Total (h) & Tempo Total (h) & Tempo Total (h) & \\
\hline 01 & Folder & 5,96 & 8,95 & 3,22 & 18,12 \\
\hline 02 & Book & 23,89 & 6,05 & 6,10 & 36,04 \\
\hline 03 & Folder & 9,92 & 0,63 & 0,53 & 11,09 \\
\hline 04 & Folder & 3,33 & 2,95 & 3,58 & 9,87 \\
\hline 05 & Folder & 3,12 & 2,92 & 0,67 & 6,71 \\
\hline 06 & Encarte & 3,67 & 13,88 & 1,45 & 19,00 \\
\hline 07 & Folder & 7,31 & 8,42 & 1,65 & 17,38 \\
\hline 08 & Folder & 16,97 & 0,97 & 1,13 & 19,07 \\
\hline 09 & Livro & 104,22 & 1,42 & 0,87 & 106,51 \\
\hline 10 & Livro & 13,70 & 8,87 & 0,00 & 22,57 \\
\hline 11 & Folder & 3,98 & 1,57 & 1,47 & 7,01 \\
\hline 12 & Revista & 4,36 & 7,78 & 7,82 & 19,96 \\
\hline 13 & Folder & 10,78 & 1,47 & 0,17 & 12,41 \\
\hline 14 & Folder & 10,33 & 2,08 & 1,25 & 13,67 \\
\hline 15 & MANUAL & 15,68 & 1,25 & 0,37 & 17,29 \\
\hline 16 & CONVITE & 32,89 & 0,98 & 0,58 & 34,46 \\
\hline 17 & TABLOIDE & 0,58 & 5,45 & 0,42 & 6,44 \\
\hline 18 & Revista & 9,39 & 1,90 & 3,27 & 14,56 \\
\hline 19 & Livro & 12,22 & 13,13 & 1,50 & 26,86 \\
\hline 20 & Revista & 58,92 & 1,47 & 1,25 & 61,64 \\
\hline 21 & Folder & 2,11 & 3,43 & 2,53 & 8,08 \\
\hline 22 & Folder & 2,89 & 2,37 & 1,68 & 6,94 \\
\hline 23 & Cartaz & 2,20 & 0,50 & 0,35 & 3,05 \\
\hline 24 & Folder & 12,22 & 1,33 & 0,75 & 14,31 \\
\hline 25 & Rótulo & 1,00 & 0,37 & 0,38 & 1,75 \\
\hline 26 & Tablóide & 3,04 & 5,08 & 4,08 & 12,21 \\
\hline 27 & Folder & 21,61 & 1,98 & 1,97 & 25,56 \\
\hline 28 & Encarte & 0,72 & 8,00 & 6,75 & 15,47 \\
\hline 29 & Lamina & 0,38 & 4,47 & 0,92 & 5,76 \\
\hline 30 & Tablóide & 0,44 & 25,58 & 0,00 & 26,03 \\
\hline & $\sum$ & 397,83 & 145,25 & 56,70 & 599,78 \\
\hline
\end{tabular}

Fonte: Pesquisa de campo (2008)

Ao final da medição, os tempos foram totalizados em função de cada um das 30 OP's e as respectivas contribuições de cada código improdutivo conforme pode ser observado na Tabela 2. Os dados mostrados na Tabela 2 foram lançados no gráfico de pareto conforme pode ser observado na Figura 4. 
Tabela 2 - Tempos improdutivos por código de improdutividade

\begin{tabular}{|c|c|c|c|c|c|c|c|c|c|c|c|c|c|c|c|c|c|c|c|}
\hline \multicolumn{20}{|c|}{ Total Geral por OP } \\
\hline OP & $\begin{array}{c}\text { Tempo } \\
\text { Total }\end{array}$ & TP & TI & cd1 & cd2 & cd3 & cd4 & cd5 & cd6 & cd7 & cd8 & cd9 & cd10 & cd11 & cd12 & cd13 & cd14 & cd15 & cd16 \\
\hline 2 & 36,04 & 11,26 & 24,78 & 0,00 & 22,31 & 0,00 & 0,00 & 0,00 & 0,00 & 0,00 & 0,00 & 0,00 & 2,47 & 0,00 & 0,00 & 0,00 & 0,00 & 0,00 & 0,00 \\
\hline 3 & 11,09 & 1,69 & 9,40 & 0,00 & 4,64 & 4,64 & 0,00 & 0,00 & 0,00 & 0,13 & 0,00 & 0,00 & 0,00 & 0,00 & 0,00 & 0,00 & 0,00 & 0,00 & 0,00 \\
\hline 4 & 9,87 & 7,37 & 2,50 & 0,00 & 0,00 & 0,00 & 0,00 & 0,00 & 2,17 & 0,00 & 0,00 & 0,00 & 0,00 & 0,00 & 0,00 & 0,00 & 0,00 & 0,00 & 0,33 \\
\hline 6 & 19,00 & 16,59 & 2,41 & 0,00 & 0,00 & 0,00 & 1,86 & 0,00 & 0,00 & 0,00 & 0,00 & 0,00 & 0,00 & 0,55 & 0,00 & 0,00 & 0,00 & 0,00 & 0,00 \\
\hline 7 & 17,38 & 4,47 & 12,91 & 0,00 & 0,00 & 6,09 & 0,00 & 0,00 & 0,00 & 0,00 & 0,00 & 0,00 & 0,00 & 6,82 & 0,00 & 0,00 & 0,00 & 0,00 & 0,00 \\
\hline 8 & 19,07 & 15,52 & 3,55 & 0,00 & 2,73 & 0,00 & 0,00 & 0,00 & 0,00 & 0,00 & 0,00 & 0,00 & 0,00 & 0,00 & 0,67 & 0,00 & 0,00 & 0,00 & 0,00 \\
\hline 9 & 106,51 & 91,97 & 14,53 & 0,00 & 14,33 & 0,00 & 0,00 & 0,00 & 0,00 & 0,00 & 0,00 & 0,00 & 0,00 & 0,00 & 0,00 & 0,20 & 0,00 & 0,00 & 0,00 \\
\hline 10 & 22,57 & 5,62 & 16,94 & 0,00 & 0,00 & 0,00 & 0,00 & 11,44 & 0,00 & 0,00 & 0,00 & 0,00 & 5,50 & 0,00 & 0,00 & 0,00 & 0,00 & 0,00 & 0,00 \\
\hline 15 & 17,29 & 2,03 & 15,27 & 0,00 & 14,33 & 0,00 & 0,00 & 0,00 & 0,00 & 0,00 & 0,00 & 0,00 & 0,00 & 0,00 & 0,00 & 0,00 & 0,00 & 0,93 & 0,00 \\
\hline 16 & 46 & 1,42 & 33,03 & 0,00 & 00 & 0,00 & 0,00 & 0,00 & 2,73 & 0,00 & 0,00 & 0,00 & 0,00 & 0,00 & 0,0 & 0,00 & 0,00 & 0,00 & 0,00 \\
\hline 17 & 6,44 & 5,42 & 1,03 & 0,00 & 0,00 & 0,00 & 0,00 & 0,00 & 0,00 & 0,24 & 0,00 & 0,00 & 0,00 & 0,00 & 0,00 & 0,00 & 0,78 & 0,00 & 0,00 \\
\hline 18 & 14,56 & 6,33 & 8,22 & 0,00 & 0,00 & 0,00 & 8,22 & 0,00 & 0,00 & 0,00 & 0,00 & 0,00 & 0,00 & 0,00 & 0,00 & 0,00 & 0,00 & 0,00 & 0,00 \\
\hline 19 & 26,86 & 5,37 & 21,49 & 0,00 & 0,00 & 0,00 & 0,00 & 0,00 & 0,00 & 11,49 & 0,00 & 0,00 & 0,00 & 0,00 & 0,00 & 0,00 & 0,00 & 0,00 & 10,00 \\
\hline 20 &, 64 & 31,78 & 29,86 & 0,00 & 29,28 & 0,00 & 00 & 0,00 & 0,00 & 0,00 & 0,00 & 0,00 & 0,00 & 0,00 & 0,00 & 0,00 & 0,00 & 0,58 & 0,00 \\
\hline 21 & 8,08 & 4,28 & 3,80 & 3,80 & 0,00 & 0,00 & 0,00 & 0,00 & 0,00 & 0,00 & 0,00 & 0,00 & 0,00 & 0,0 & 0,00 & 0,00 & 0,00 & 0,00 & 0,00 \\
\hline 22 & 694 & 5,62 & 1,32 & 0,00 & 0,00 & 0,00 & 0,00 & 0,00 & 0,00 & 0,00 & 1,32 & 0,00 & 0,00 & 0,0 & 0,0 & 0,00 & 0,00 & 0,00 & 0,00 \\
\hline 23 & 3,05 & 1,27 & 1,78 & 0,00 & 0,00 & 0,00 & 0,00 & 0,00 & 0,00 & 1,61 & 0,00 & 0,00 & 0,00 & 0,00 & 0,00 & 0,00 & 0,00 & 0,17 & 0,00 \\
\hline 24 & 14,31 & 2,56 & 11,75 & 0,00 & 0,00 & 0,00 & 0,00 & 0,00 & 0,00 & 0,00 & 11,67 & 0,00 & 0,08 & 0,00 & 0,00 & 0,00 & 0,00 & 0,00 & 0,00 \\
\hline$\%$ & & $51 \%$ & $49 \%$ & $1,2 \%$ & $14,8 \%$ & $3,3 \%$ & $6,1 \%$ & $2,4 \%$ & $6,7 \%$ & $2,5 \%$ & $2,7 \%$ & $0,0 \%$ & $2,0 \%$ & $2,9 \%$ & $0,1 \%$ & $0,6 \%$ & $0,9 \%$ & $0,5 \%$ & $1,7 \%$ \\
\hline
\end{tabular}

Fonte: Pesquisa de campo (2008)

Figura 4 - Participação dos tempos improdutivos de cada código para o tempo improdutivo total

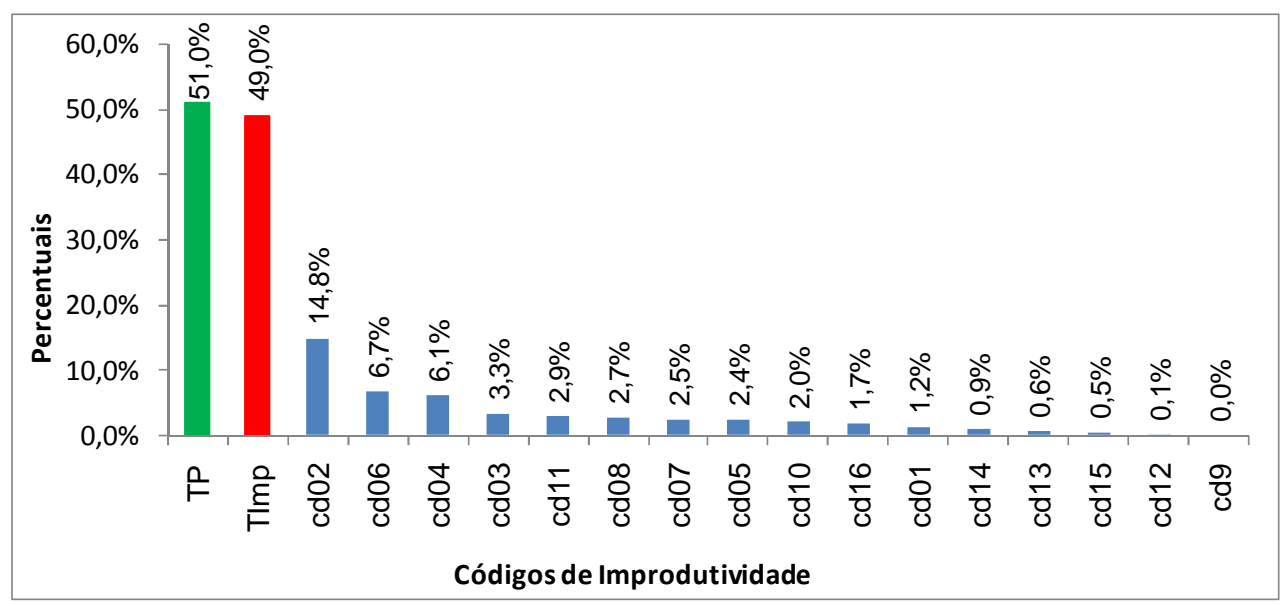

Fonte: Autoria própria (2009) 
A partir da análise deste gráfico verifica-se que boa parte do tempo é consumido nas interrupções, cerca de $49 \%$ do tempo total. O gráfico permite ainda verificar a contribuição de cada código de improdutividade para a composição do tempo improdutivo.

\section{Construção do modelo matemático regressivo (MMR)}

Para construção do MMR determinaram-se quais códigos seriam significativos para a construção do mesmo, no intuito de simplificar a equação do tempo improdutivo total a partir da redução de variáveis com menor impacto na variação desse tempo. Para tanto, após realizado o teste de regressão Stepwise, observou-se que os códigos cd9 - "Máquina em manutenção" e cd 12 "Reuniões e treinamentos" deveriam ser desconsiderados do modelo. Tais códigos apresentaram valores de frequiência iguais a zero e $0,1 \%$, respectivamente, conforme pode ser visto no gráfico da figura 4. Dessa forma demonstraram ser pouco significativos visto que tais eventos ocorrem com um nível de freqüência muito baixo, sendo, portanto valores com baixa significância para o modelo.

Com relação ao código cd 15 - “aguardando plotter”, apesar de ter um percentual pouco expressivo, na prática contribuía para ociosidade dos funcionários, demandando tempo de esperar à conclusão do serviço em andamento, impactando em processo de filas. Para sanar tal problema foi adquiriu-se um plotter de qualidade eficaz.

Após feita a triagem das variáveis menos significativas, parte-se para a definição do modelo representado pela equação obtida a partir do tratamento estatístico mediante uso de regressão linear múltipla, através de software estatístico. O resultado da análise de regressão nos fornece o modelo regressivo tempo improdutivo (TI).

$$
T I=1,62+0,973 c d 2+0,827 c d 3+0,904 c d 4+1,28 c d 5+0,949 c d 6+0,883 c d 8+0,871 c d 10+1,98 c d 15
$$

Tem-se que esse modelo é consistente tendo em vista que $F=98,02$ e p=0,000<0,005; e para cada valor de $t$ _student correspondente a cada parâmetro da equação acima, $\mathrm{p}<0,005$, o que dá credibilidade à existência das variáveis no modelo.

\section{Conclusões}

Com a identificação dos fatores que contribuem para elevar o tempo improdutivo do departamento de pré-impressão, é possível estabelecer meios de mantê-los sob controle, de tal forma a prevenir situações e preparar o pessoal do setor para mudanças que visem o aumento na produtividade do departamento.

O MMR apresentado possibilitou ao gerente de produção constatar que $96,4 \%$ da variação 
do tempo improdutivo foram devidas às variáveis códigos improdutivos. Ou seja, aguardar a chegada de um plotter, treinamentos, reuniões, máquinas em manutenção e outros fatores possibilitam aumento representativo de tempos ociosos no processo de preparação das chapas de impressão. Dessa constatação o gerente de produção poderá tomar decisões de forma mais precisas.

O MMR mostra que a espera por um plotter tem um impacto de $1,98 \mathrm{t}$ na queda de produtividade, pois promove formação de filas e atraso na programação do PCP. Para sanar esse problema adquiriu-se mais uma plotter para o setor, distribuindo assim melhor as ordens de produção. Os problemas com lombada possuem 1,28t de impacto; os problemas no formato do arquivo com representatividade de $0,97 \mathrm{t}$ e ausência de projeto com $0,94 \mathrm{t}$, para os quais foram adotadas a utilização de check lists que auxiliam no cumprimento dessas atividades com o mínimo erro possível.

Tais informações como outras fornecidas por esse MMR podem auxiliar medidas eficazes para tomada decisões, revelando que o controle dessas variáveis poderá reduzir o tempo improdutivo no processo do sistema de produção de pré-impressão dessa empresa gráfica.

A partir da definição das variáveis mais representativas para o tempo improdutivo pode-se precisar, com um nível de confiança aceitável, onde intervir para minimizar os problemas relacionados à produtividade do sistema.

\begin{abstract}
This article discusses a study in the department of Pre-printing (IP) of a graphic, with the objective of verifying the influence of the variable processing time of the activities in the department for the preparation of plates for printing. The results may provide support for decision making. Thus, it was used for graphical modeling and multivariate Pareto from the treatment of 30 orders of production. It was concluded that about $49 \%$ of the total processing time is bound to be bottlenecks in the system, and $96.4 \%$ of the variation of the time were due to unproductive idleness existing during the preparation of plates for printing.
\end{abstract}

Key-words: the regression analysis; processing time; pre-press

\title{
Referências
}

ALMEIDA FILHO, Benicio Ferreira de; FERREIRA, João Roberto; PAIVA, Anderson Paulo de. Análise de Regressão e Simulação na avaliação de Tempos em Processo de Usinagem Pesada. In: XXVIII Encontro Nacional de Engenharia de Produção, Rio de Janeiro, 2008.

ARAGÃO, Irlam Reis de. A redução de perdas num processo produtivo através da implantação da sistemática da árvore de perdas. Revista Produção, v. 7, n. 2, Ago. 2008: Florianópolis, SC.

FILHO, J.T.A. A customização em massa na construção civil: um estudo no setor de edificações. Dissertação de Mestrado do Programa de Pós-Graduação em Engenharia de Produção da Universidade federal da Paraíba, João Pessoa, 2008 .

MACHADO, A.G.C.; MORAES, W.F.A. Estratégias de customização em massa implementadas por empresas brasileiras. Revista Produção, v. 18, n. 1, p. 170-183, Jan./Abr. 2008.

PINCOLINI, Maria Medianeira. A análise de regressão aplicada na comparação do peso de recém-nascidos. In: $49^{a}$ Reunião da RBRAS. Uberlândia: UFU/MG. Maio, 2004 
SANTOS, Adriana de Paula Lacerda; MENDES JÚNIOR, Ricardo. Simulação de processos de construção como ferramenta de melhorias. In: Seminário de Tecnologia da Informação e Comunicação na Construção Civil. Curitiba: UFPR, 2003.

SANTOS, Alcione Miranda dos. Análise de Regressão - Aula 2. Departamento de Saúde Pública. Universidade Federal do Maranhão: UFMA, 2003.

SLACK, Nigel; CHAMBERS, Stuart; JOHNSTON, Robert. Administração da Produção. 2. Ed. São Paulo: Atlas, 2002.

GAITHER, Norman; FRAZIER, Greg. Administração da produção e operações. 8. Ed. São Paulo: Cengage Learning, 2008.

OHNO, Taiichi. O sistema Toyota de produção além da produção em larga escala. Porto Alegre: Bookman, 1997

ORGANIZACIÓN INTERNACIONAL DEL TRABAJO - OIT. Introducción al estúdio del trabajo. Genebra. Oficina internacional del trabajo, 1994.

\section{DADOS DOS AUTORES}

Nome completo: Márcio Carvalho da Silva

Filiação institucional: Universidade Federal da Paraíba - UFPB

Departamento: Programa de pós-graduação em Engenharia de Produção - UFPB

Função ou cargo ocupado: Mestrando

Endereço completo para correspondência (bairro, cidade, estado, país e CEP): Rua Projetada, 92 -

Ponta de Campina - Cabedelo - Paraíba - Brasil - CEP: 58310-000

Telefones para contato: (83) 8894-8462

e-mail:mkarvalho2005@yahoo.com.br

\section{Nome completo: Maria de Lourdes Gomes Barreto}

Filiação institucional: Universidade Federal da Paraíba - UFPB

Departamento: Programa de pós-graduação em Engenharia de Produção - UFPB

Função ou cargo ocupado: Professora Permanente

Endereço completo para correspondência (bairro, cidade, estado, país e CEP): Centro de Tecnologia da UFPB - Campus I - Cidade Universitária - João Pessoa - Paraíba - Brasil - CEP: 58051-970

Telefones para contato: (83) 9986-6509

e-mail:marilu@ct.ufpb.br 


\section{Nome completo: Luis Bueno da Silva}

Filiação institucional: Universidade Federal da Paraíba - UFPB

Departamento: Programa de pós-graduação em Engenharia de Produção - UFPB

Função ou cargo ocupado: Professor Permanente

Endereço completo para correspondência (bairro, cidade, estado, país e CEP): Centro de Tecnologia da UFPB - Campus I - Cidade Universitária - João Pessoa - Paraíba - Brasil - CEP: 58051-970

Telefones para contato: (83) 9106-6287

e-mail:bueno@ct.ufpb.br 